\title{
RELACIONES ENTRE LA ESTRUCTURA COGNITIVA Y LA INTENSIDAD DE LA SINTOMATOLOGÍA EN DOS SUBGRUPOS DE MUJERES MALTRATADAS: UN ESBOZO DE TIPOLOGÍAS DE LAS VÍCTIMAS DE LA VIOLENCIA CONTRA LA PAREJA
}

\section{RELATIONS BETWEEN THE COGNITIVE STRUCTURE AND THE INTENSITY OF THE SYMPTOMATOLOGY IN TWO BATTERED WOMEN'S SUBGROUPS. AN OUTLINE OF TYPOLOGIES OF BATTERED WOMEN}

\author{
JESÚS GARCIA-MARTÍNEZ \\ Ma CARMEN ORELlanA-RAmíREZ² \\ RAFAEL GUERRERO-GÓMEZ ${ }^{3}$ \\ 1(jgm@us.es) 2(mcorellana@us.es) Departamento de Personalidad, Evaluación \\ y Tratamiento Psicológicos. Universidad de Sevilla. \\ 3(copsica@yahoo.es). Centro de Orientación y Psicología «Cerro del Águila».Sevilla.
}

Cómo referenciar este artículo/How to reference this article:

García-Martínez, J., Orellana-Ramírez, M. C. y Guerrero-Gómez, R. (2012). Relaciones entre la estructura cognitiva y la intensidad de la sintomatología en dos subgrupos de mujeres maltratadas: un esbozo de tipologías de las víctimas de la violencia contra la pareja [Relations between the cognitive structure and the intensity of the symptomatology in two battered women's subgroups. An outline of typologies of battered women]. Acción Psicológica, 9(1), 47-60. doi: http://dx.doi.org/10.5944/ap.9.1.436

\section{Resumen}

Se evalúa la sintomatología de una muestra de mujeres víctimas de violencia de la pareja $(n=24)$ con el SCL-90-R y los inventarios de Depresión y Ansiedad de Beck. La estructura cognitiva es evaluada utilizando la técnica de rejilla y se tienen en cuenta las siguientes variables: polaridad, potencia, intensidad, indefinición, constructos funcionalmente independientes, va- rianza explicada por el primer factor, presencia de dilemas y correlaciones entre facetas del yo. La muestra fue dicotomizada en función de los puntos de corte de los instrumentos que evalúan sintomatología. Los resultados indican que las mujeres con sintomatología más severa obtienen una mayor indefinición y una menor complejidad en términos de constructos independientes. Las mujeres que presentan dilemas obtienen valores más altos en porcentaje de va-

Agradecimientos: esta investigación se llevó a cabo gracias al convenio financiado por la Diputación Provincial de Sevilla y coordinado por la Universidad de Sevilla durante la anualidad 2007-2008. 
rianza explicada por el primer eje y muestran una mayor sensibilidad interpersonal. La combinación de dilemas y sintomatología intensa parece presentar el peor pronóstico.

Palabras Clave: Violencia contra la pareja; técnica de rejilla; sintomatología; tipologías de mujeres maltratadas

\section{Abstract}

The symptoms of a simple sample of women victims of intimate partner violence $(n=24)$ are assessed using the SCL-90-R and the Beck's Depression and Anxiety Inventories. The cognitive structure is assessed with the repertory grid technique. The variables taken in account were polarization, power, intensity, ambiguity, presence of dilemmas and correlations between self facets. Sample was divided using the clinical cuts points of the symptoms instruments. Results show that women with higher symptomatology got higher values in ambiguity and lower in functionally independent constructs. Women with dilemmas show a higher value in variance explained by first axis and in interpersonal sensibility. Combination of dilemmas and higher symptomatology is the profile with a worse prognosis.

Key concepts: intimate partner violence; repertory grid technique; symptoms; typologies of battered women

\section{Introducción}

La violencia contra las mujeres afecta a todas las clases sociales, todos los niveles educativos y todos los entornos geográficos, por ello representa un gran problema social y psicológico que tiene que afrontar el conjunto de la sociedad. Se trata de una forma de violencia muy extendida en todo el mundo y es la más común de las violencias interpersonales en los países desarrollados (Fischbach y Herbert, 1997; Krug, Dahlberg, Mercy, Zwi y Lozano, 2002), en los que se calcula que como mínimo el 10\% de las mujeres se ven afectadas por la misma (Römkens, 1997). Dada su altísima prevalencia (es mayor que la de la mayor parte de las enfermedades orgánicas más comunes) se debe considerar un grave problema de salud pública (Heise y García Moreno, 2002). Las repercusiones psicológicas del maltrato y el abuso físico constituyen un factor de riesgo de salud a largo plazo (Koss, Koss y Woodruff, 1991; Matud, 2004).

No hay evidencia de que haya un perfil prodrómico de la mujer víctima, más bien los procesos que llevan a la victimización se basan en una serie de ciclos de violencia (Dutton y Painter, 1981; Walker, 2004) en los que el agresor va progresivamente controlando la conducta y la visión del mundo de la víctima. A pesar de que en el caso de la violencia contra la pareja los criterios de control están basados en supuestos de género, la violencia se usa para mantener un status quo de desigualdad (Jacobson y Gottman, 1998), aspecto que comparte éste tipo de violencia con otras violencias interpersonales (Garcia-Martínez, 2008).

A lo largo del proceso de control por parte del agresor se van manifestando distintos tipos de sintomatología psicológica que incluyen la caída de la autoestima y la autoeficacia (Orava, McLeod y Sharpe, 1996), así como sentimientos de culpa, aislamiento social y dependencia del maltratador (Buchbinder y Eisikovits, 2003; Echeburúa y de Corral, 1998). El maltrato es un factor que genera trastornos y síntomas psicopatológicos de varios tipos como depresión, estrés postraumático, baja autoestima, sentimientos de culpa, etc. (Echeburúa y de Corral, 1998; Golding, 1999). De las distintas investigaciones realizadas se concluye que los síntomas más usuales padecidos por las mujeres maltratadas se agrupan en dos bloques: uno ligado a la depresión y la ansiedad que incluiría, en su caso, el déficit de autoestima; y otro, menos frecuente, relacionado con el estrés postraumático.

Pero hay pocos estudios que relacionan la sintomatología en términos directos con la forma en que las mujeres maltratadas estructuran su sistema cognitivo y cómo intentan dar sentido a la situación que atraviesan (Camps, Calle y Feixas, 2000; Horley y Johnson, 2008). Desde el punto de vista terapéutico, entender el modo en que se estructura el sistema cognitivo puede ser algo básico para dirigir las intervenciones con las víctimas, ya que facilitará diseñar procesos terapéuticos que sean realmente útiles. 
En este sentido, el modelo de construcción personal (Kelly, 1955) es quizá uno de los modelos teóricos que proporcionan más herramientas conceptuales y de evaluación para estudiar la estructura cognitiva. El fundamento básico de esta perspectiva es que los seres humanos desarrollan a partir de su experiencia una serie de dimensiones individuales de tipo bipolar que les sirven tanto para describir la realidad como para regular la acción. A esas dimensiones se les denomina constructos. Estos constructos son muy variables en su contenido, aplicación y rango de conveniencia y están regulados por una serie de leyes o corolarios que explican su funcionamiento. El modo en el que se aplican los constructos determina la estructura cognitiva del individuo.

Desde el punto de vista de la evaluación, una de las herramientas más conocidas del modelo de constructos personales es la técnica de la rejilla (Feixas y Cornejo, 1996; Fransella, Bell y Bannister, 2004; Kelly, 1955). Se trata de una entrevista muy peculiar en la que el/a evaluador/a elige una serie de elementos a partir de los cuáles se va a obtener información del cliente. Estos elementos se eligen por razones de pertinencia y relevancia para el tema a explorar y suelen ser aspectos del self (self actual, self ideal, self después de un cierto cambio), síntomas o problemas (la relación con el padre, la fobia, la enfermedad), o personas relevantes para el sujeto (pareja, padre, madre). Los elementos se comparan entre sí bien en tríos (procedimiento triádico) o en parejas (procedimiento diádico) y se pregunta al sujeto en qué aspectos psicológicamente relevantes se parecen y se diferencian. De ese contraste entre semejanza y diferencia surge un constructo. La administración de la rejilla finaliza cuando la persona no es capaz de generar nuevos constructos o cuando éstos se repiten. Si la rejilla se aplica en un contexto de investigación, el criterio de cese es la obtención de una rejilla con tantos constructos como elementos. Una vez finalizada la rejilla, todos los constructos se puntúan para todos los elementos utilizando para ello una escala de intervalo.

La aplicación de la rejilla al trabajo con mujeres víctimas de la violencia de pareja se ha centrado en algunas dimensiones estructurales de la rejilla como la polaridad o la complejidad del sistema de construcción (Camps et al., 2000; Guerrero-Gómez, Garcia-Martínez y OrellanaRamírez, 2009; Soldevilla, Feixas, Varlotta, Cirici y Ayats, 2009 ), la diferencia entre las construcciones del yo y el ideal (Harter, 2000; Soldevilla et al., 2009) o la construcción de los roles de género (Garcia-Martínez, Orellana-Ramírez y Guerrero-Gómez, 2010). Otra variable que se ha incluido en estos trabajos es la presencia de dilemas cognitivos como elementos característico de las víctimas (Garcia-Martínez et al. 2009; Soldevilla et al., 2009).

En este artículo se continúa esta línea de investigación poniendo especial énfasis en aspectos objetivos de la sintomatología, de la identidad (medidos mediante la rejilla) y la relación entre ambas. Entendemos que aunar ambas perspectivas científicas (psicométrica y cualitativa-constructivista) es imprescindible para avanzar en el estudio y el trabajo terapéutico del complejo y dramático fenómeno de la violencia contra la pareja.

El objetivo básico de este estudio es encontrar las relaciones entre la estructura de la construcción personal y la sintomatología que manifiestan mujeres que han sufrido violencia contra la pareja íntima. Dado que en una investigación anterior nos hemos centrado en cuestiones relacionadas con la presencia-ausencia de dilemas y la complejidad de la construcción (Garcia-Martínez et al., 2009; Soldevilla et al., 2009) se establecen, para el presente estudio, tres hipótesis:

1. Las mujeres con mayor intensidad de la sintomatología mostrarán un sistema más rígido (mayores puntuaciones en polaridad total).

2. Las mujeres con mayor intensidad de la sintomatología mostrarán un sistema más simple (mayores valores en la varianza explicada por el primer eje y menores valores en el número de constructos funcionalmente independientes).

3. Las mujeres con mayor intensidad de la sintomatología presentarán más dilemas implicativos.

No obstante, dado que se sabe relativamente poco del comportamiento de otros indicadores 
estructurales de la rejilla en relación con casos de violencia y, en concreto, en casos de violencia contra la pareja íntima, el análisis exploratorio abarcará variables como las correlaciones entre el yo-ideal-otros, la potencia, la intensidad y el índice de indefinición.

\section{Método}

\section{Participantes}

La muestra fueron 24 mujeres de localidades de pequeño tamaño de la Provincia de Sevilla (entre 500 y 19000 habitantes). Todas ellas fueron remitidas por las abogadas de los Puntos de Información a la Mujer de sus municipios. Todas se encontraban separadas o en proceso de separación debido a violencia de género y demandaron explícitamente ayuda terapéutica. La edad media era de 43.81 años $(S D=10.84)$. Presentaban un perfil socioeconómico bajo. El $7.7 \%$ eran analfabetas funcionales, el $30.8 \%$ tenían estudios primarios incompletos, el 38.5\% estudios primarios completos, el $7.7 \%$ tenían estudios secundarios, y el $11.75 \%$ estudios postsecundarios; ninguna tenía titulación universitaria superior. En cuanto a la ocupación profesional, el 73\% estaban en activo; el 7.7\% eran exclusivamente amas de casa y el $11.5 \%$ eran pensionistas. Entre los trabajos más usuales, destacaban las labores agrícolas (23.1\%), empleos de bajo nivel en el sector público (19.2\%) y la industria de transformación agraria (15.4\%).

\section{Instrumentos}

Para valorar la sintomatología se han empleado las versiones españolas del Inventarios de Ansiedad (IAB, Beck, Brown, Epstein y Steer, 1988) y Depresión de Beck (IDB, Beck, Rush, Shawn y Emery, 1979) y del Cuestionario de Síntomas SCL-90-R (Derogatis, 2000). Los dos inventarios de Beck son dos escalas que evalúan una única dimensión (ansiedad o depresión) y constan de 21 ítems con un intervalo de respuesta entre 0 y 3 . El cuestionario de Derogatis evalúa hasta 9 escalas sintomatológicas distintas (somatización -SOM-, Obsesión-OBS-, Sensi- bilidad Interpersonal -SIP-, Ansiedad -ANS-, Miedo-Hostilidad -MH-, Ansiedad Fóbica AFOB-, Ideación Paranoide -IPAR- y Psicoticismo -PSI-) y cuenta con tres indicadores globales de patología, el índice global de síntomas (IGS), el total de síntomas positivos (TSP) y el Índice de Malestar Sintomático Positivo (IMSP). Consta de 90 ítems que se distribuyen desigualmente entre las distintas escalas y el intervalo de respuesta es de 0 a 4 .

Los síntomas de carácter afectivo son los más usuales en los casos de víctimas de violencia contra la pareja íntima (Amor, Echeburúa, de Corral, Zubizarreta y Sarasua, 2001; Matud, 2004) por lo que es conveniente evaluarlos de modo explícito y directo (IAB e IDB). El uso de una prueba de rastreo de sintomatología como el SCL-90 ofrece un amplío rango de detección de problematicidad psicopatológica sin la necesidad de efectuar un diagnóstico explícito.

Por otro lado, la estructura cognitiva se evalúa con la rejilla típica del proyecto multicéntrico dilema (Feixas y Saúl, 2004): una rejilla cuadrada de 15 elementos prácticamente predeterminados pero que se modifican ligeramente para adaptarlos a cada investigación concreta. El procedimiento de obtención de constructos es el diádico y la escala de intervalo utilizada para puntuar los constructos a través de los elementos ha sido de 7 puntos (1 representa el máximo valor del polo de la izquierda, 7 el máximo valor del polo de la derecha y 4 una puntuación intermedia entre ambos). Por ejemplo: si el constructo es inteligente-perspicaz (recuérdese que los constructos son personales $\mathrm{y}$, por tanto, los contrastes entre polos no tienen por qué seguir contraposiciones semánticas ni lógicas), una persona con una puntuación de 7 sería muy perspicaz, pero no sería inteligente; una persona con una puntuación de 2 sería bastante inteligente, pero no sería perspicaz; alguien con una puntuación de 4 no sería ni inteligente ni perspicaz (o bien ambas cosas a la vez y con la misma intensidad).

Los elementos elegidos para la construcción de la rejilla han sido: yo actual, yo antes de tener conciencia de ser una mujer maltratada, yo dentro de 6 meses, pareja que la maltrataba, padre, madre, familiar relevante (a elegir por la mujer), mujer feliz, mujer infeliz, varón que trata bien 
a los demás, varón que trata mal a los demás, persona significativa 1 , persona significativa 2 , terapeuta y yo ideal. La mujer identificó dentro de sus relaciones cercanas a las personas que desempeñaban todos los papeles. La rejilla se administró siguiendo el procedimiento de comparaciones diádicas (comparando parejas de elementos preseleccionadas) en la sesión tres o cuatro de terapia. El catálogo de comparaciones diádicas estaba preestablecido en todos los casos. La administración de la rejilla cesaba cuando se producía el constructo 15 . $^{\circ}$.

\section{Medidas}

Para valorar la psicopatología se utilizan las siguientes medidas: puntuación absoluta de las distintas variables del SCL-90-R y del IAB e IDB.

En la rejilla se han utilizado las siguientes medidas:

1. Variables relativas a la estructura del sistema de construcción son:

a) Construcciones funcionalmente independientes (CFI). Es un indicador del número de elementos y constructos que están agrupados entre sí e indica el nivel de complejidad de la construcción. Operativamente es el número de agrupaciones funcionales dividido por la suma total de elementos y constructos de la rejilla. Las agrupaciones funcionales son el número de conglomerados de constructos o elementos que aparecen en función de las correlaciones entre ellos. Es un dato generado por el programa informático de análisis Record (Feixas y Cornejo, 2002). En nuestro caso: $\mathrm{x} / 30$. Cuanto mayor es el valor, mayor es la complejidad.

b) Porcentaje de Varianza Explicada por el Primer Eje (PVEPE). Es el valor de la principal dimensión de significado, producto de un análisis de correspondencias simples previo. Cuanto mayor es el valor de esa dimensión, menor es el número de componentes relevantes que se utilizan de la construcción de la experiencia. Teniendo en cuenta que los ejes representan dimensiones de significado, el va- lor de la varianza explicada del primer eje representa la magnitud de la principal dimensión de significado. Se considera el mejor indicador de la complejidad. Si el valor es alto, nos indica que el sujeto explica mucho acerca de su mundo en un solo eje, con lo cual podemos entender una cierta forma de construir su mundo «unidimensional». Si la puntuación es baja, estaríamos ante el caso de un sujeto con una mayor complejidad cognitiva (Feixas y Cornejo, 1996). En el caso del programa Record (Feixas y Cornejo, 2002), los datos se agrupan siempre en cinco ejes ordenados de mayor a menor en función del porcentaje de varianza explicada.

c) Polaridad. Este índice muestra el grado en el que el sujeto utiliza puntuaciones extremas (1 ó 7). Se calcula dividiendo el número de puntuaciones extremas entre el total de constructos. Las puntuaciones altas se consideran una forma de rigidez cognitiva. Al contrario, las puntuaciones bajas nos dan una idea de la laxitud cognitiva del sujeto (Feixas y Cornejo, 1996).

d) Indefinición. Es el indicador opuesto a la polaridad. El porcentaje de valores no extremos usados por el sujeto. Indica la incapacidad para definir de forma precisa la naturaleza de una construcción dada.

e) Intensidad. Habitualmente se considera una medida de la integración, es decir, del grado en que los constructos se asocian o enlazan entre sí (Bannister y Fransella, 1966). En el fondo, no obstante, es un indicador de la multidimensionalidad de la construcción, ya que su cálculo se basa en las correlaciones entre un constructo y la media de los demás (Feixas y Cornejo, 1996). A mayor valor, mayor es la intensidad.

2. Potencia. Es una medida de la sutilidad de la aplicación de los constructos, es decir, del grado de precisión o detalle con que un constructo se aplica a los diferentes elementos. Su cálculo tiene en cuenta la cantidad de 
puntuaciones distintas utilizadas (Feixas y Cornejo, 1996).

3. Variables relacionadas con la construcción del self. Se trata de correlaciones Pearson entre actual self, ideal self y el elemento-contenedor others. Otros es el resultado de la media de todos los elementos no relacionados con el yo (Feixas y Cornejo, 1996). Estas correlaciones permiten estudiar la autoestima del sujeto, siguiendo estos criterios:

a) Correlación yo actual-yo ideal: es una medida de la autoestima directa del sujeto, mide el grado de proximidad entre la autopercepción del sujeto y su imagen ideal.

b) Correlación yo actual-otros. Es el grado de proximidad entre la autopercepción y la percepción de los otros. Evalúa el grado de aislamiento social percibido (cuanto mayor sea el valor, menor es el aislamiento).

c) Correlación ideal-otros. Es el grado de proximidad entre la imagen ideal o meta personal y la percepción de los otros. Evalúa el grado en que otros se aproximan a la idealización, lo que implica el grado de adecuación atribuido a los demás.

4. Dilema Implicativo (dilema). Una última variable considerada en nuestro análisis es la presencia del dilema implicativo. El dilema implicativo es la evidencia de una incoherencia en el sistema de construcción del sujeto, lo que supone que la persona va a tener dificultades en la elaboración y desarrollo de su conducta cuando para llevar a cabo una actividad tenga que utilizar simultáneamente los dos constructos implicados en el dilema.

Para entender la noción de dilema, hay que distinguir entre constructos congruentes y constructos discrepantes (Feixas, Saúl y Ávila, 2009; Feixas, Saúl, Ávila y Sánchez 2001). Los primeros son aquellos en que la posición del actual self coincide con la del ideal self, de modo que la persona ubica ambos aspectos del self en el mismo polo del constructo y con una intensidad similar. Los segundos son aquellos en que la posición del actual self y el ideal self diverge, de modo que el actual self está ubicado en un polo distinto al ideal self. Matemáticamente se define el constructo discrepante como aquel en que la diferencia entre el valor otorgado al self ideal y al actual self es al menos de cuatro puntos absolutos. Por ejemplo, en el constructo inteligente-hábil, si actual self tuviera un valor de 2, pero ideal self de 7, se obtendría la siguiente diferencia, $2-7=-5$. El valor 5 indicaría que este constructo es discrepante para el sujeto.

El dilema implicativo se produce cuando se activan simultáneamente un constructo congruente y otro discrepante y además éstos están correlacionados positivamente entre sí, de manera que un cambio en un constructo (un cambio de ubicación del actual self entre los polos del constructo) conlleva un cambio en el otro (también un cambio de ubicación del actual self entre los polos).

\section{Procedimiento}

El proceso de terapia y recogida de datos se llevó a cabo en la localidad de origen de las mujeres.

Las pruebas psicométricas fueron administradas al final de la primera sesión y la rejilla en la sesión $3 .^{\mathrm{a}}$ ó $4 .^{\mathrm{a}}$ de la terapia. La rejilla se corrigió usando el programa Record 4.0 (Feixas y Cornejo, 2002). Las puntuaciones de sintomatología fueron divididas en dos categorías dicotómicas: por encima y por debajo de valores de significación clínica, que están establecidos en los siguiente valores: 169 para el IGS (Derogatis, 2002), 19 para el IAB (Beck, Brown, Epstein y Steer, 1988) y 16 para el IDB (Bobes, García-Portilla, Bascarán, Sáiz y Bousoño, 2004).

El diseño estadístico fue de comparación entre grupos. Dado el bajo $N$ de la muestra se utilizaron pruebas no paramétricas. Los análisis estadísticos se realizaron con el programa SPSS 16.0 .

\section{Diseño}

Análisis exploratorio ex post facto. 
Tabla 1

Valores de las variables estructurales de la rejilla en relación con la sintomatología

\begin{tabular}{|c|c|c|c|c|c|c|c|c|c|c|}
\hline Síntomas & Estadísticos & $\begin{array}{l}\text { Intensi- } \\
\text { dad }\end{array}$ & Potencia & Polaridad & $\begin{array}{l}\text { Indefini- } \\
\text { ción }\end{array}$ & CFI & $\begin{array}{l}\text { R Yo- } \\
\text { Ideal }\end{array}$ & $\begin{array}{l}\text { R Yo- } \\
\text { Otros }\end{array}$ & $\begin{array}{c}\mathbf{R} \\
\text { Otros- } \\
\text { Ideal }\end{array}$ & PVEPE \\
\hline \multirow{4}{*}{$\begin{array}{l}\text { IGS-SCL } \\
\text { Dicotomizado } \\
\text { N Altos: } 13 \\
\text { N Bajos } 11\end{array}$} & U & 42.500 & 45.000 & 47.500 & 23.000 & 20.000 & 41.000 & 42.000 & 49.000 & 43.000 \\
\hline & z & -1.182 & -1.018 & -.854 & -2.467 & -2.666 & -1.280 & -1.215 & -.755 & -1.149 \\
\hline & P Asintótica & .237 & .309 & .393 & .014 & .008 & .200 & .224 & .450 & .250 \\
\hline & P Exacta & .243 & .332 & .401 & .013 & .007 & .217 & .243 & .478 & .270 \\
\hline \multirow{4}{*}{$\begin{array}{l}\text { IDB } \\
\text { Dicotomizado } \\
\text { N Altos: } 19 \\
\text { N Bajos } 5\end{array}$} & U & 42.500 & 45.000 & 47.500 & 23.000 & 20.000 & 41.000 & 42.000 & 49.000 & 43.000 \\
\hline & Z & -1.182 & -1.018 & -.854 & -2.467 & -2.666 & -1.280 & -1.215 & -.755 & -1.149 \\
\hline & P Asintótica & .237 & .309 & .393 & .014 & .008 & .200 & .224 & .450 & .250 \\
\hline & P Exacta & .243 & .332 & .401 & .013 & .007 & .217 & .243 & .478 & .270 \\
\hline \multirow{4}{*}{$\begin{array}{l}\text { IAB } \\
\text { Dicotomizado } \\
N \text { altos: } 20 \\
\text { N bajos: } 4\end{array}$} & U & 37.000 & 28.000 & 33.000 & 27.500 & 37.500 & 28.000 & 27.000 & 24.000 & 28.000 \\
\hline & Z & -.232 & -.930 & -.542 & -.970 & -.194 & -.930 & -1.007 & -1.239 & -.930 \\
\hline & P Asintótica & .816 & .353 & .588 & .332 & .846 & .353 & .314 & .215 & .353 \\
\hline & P Exacta & .852 & .388 & .627 & .347 & .852 & .388 & .347 & .241 & .388 \\
\hline
\end{tabular}

\section{Resultados}

\section{Relaciones entre sintomatología y sistema de construcción}

Una vez clasificada la muestra en función de los puntos de corte clínicos, se aplicó el estadístico U de Mann-Whitney a todas las variables relacionadas con la estructura del sistema de constructos y con la construcción del self (correlaciones self-ideal-otros). Los resultados se detallan para la distribución a través de los tres indicadores de psicopatología utilizados (Índice Global de Síntomas del SCL-90-R; IAB e IDB).

En la tabla 1 se puede ver que solamente dos variables de la rejilla son sensibles a la intensidad de la sintomatología, el índice de indefinición y el número de constructos funcionalmente independientes. Estas dos variables producen diferencias significativas para dos de los indicadores patológicos, la dicotimización del índice general de síntomas del SCL y la del Inventario de Depresión de Beck. Los valores de los rangos promedios de los grupos bajo y alto en el caso del IGS son para la indefinición 14.91 y 9.09 (grupo bajo: $\mathrm{M}=11.919, \mathrm{SD}=11.662$; grupo alto: $\mathrm{M}=5.060, \mathrm{SD}=5.951)$. Para los CFI, son 15.08 (grupo bajo: $\mathrm{M}=.474, \mathrm{SD}=.229$ ) y 7.81 (grupo alto: $\mathrm{M}=.313, \mathrm{SD}=.184$ ). Los rangos promedios de la dicotomización del IDB son en el caso de la indefinición 14.30 para el grupo bajo $(\mathrm{M}=8.088, \mathrm{SD}=7.037)$ y 12.03 para el grupo alto $(M=8.234, S D=19.185)$; para el $C F I$ los rangos son 15.20 (grupo bajo: $\mathrm{M}=.391, \mathrm{SD}$ $=.102)$ y 11.79 (grupo alto: $\mathrm{M}=.385, \mathrm{SD}=.241$ ). Para el inventario de ansiedad de Beck no se generan diferencias significativas.

\section{Relaciones entre dilemas, sintomatología y sistema de construcción}

Se utilizó un sistema similar de dicotomización de la muestra en función de la presencia o ausencia de dilemas para comprobar las relaciones entre éstos, la intensidad de la sintomatología y los valores de otras variables de la 
Tabla 2

Sintomatología en función de la presencia $(n=13)$-ausencia $(n=11)$ de dilemas en la rejilla

\begin{tabular}{lcccccccccccccc}
\hline $\begin{array}{l}\text { Estadís- } \\
\text { ticos }\end{array}$ & SOM & OBS & SIP & DEP & ANS & M-H & AFOB & IPAR & PSI & IGS & TSP & IMPD & IDB & IAB \\
\hline U & 58.500 & 54.500 & 25.000 & 47.000 & 52.000 & 42.000 & 60.000 & 31.500 & 54.500 & 58.000 & 59.000 & 55.000 & 52.500 & 57.500 \\
$Z$ & -.132 & -.395 & -2.340 & -.888 & -.559 & -.917 & -.033 & -1.908 & -.395 & -.164 & -.099 & -.361 & -1.103 & -.812 \\
$\begin{array}{l}P \text { asin- } \\
\text { tótica }\end{array}$ & .895 & .693 & $.019^{*}$ & .375 & .576 & .359 & .974 & .056 & .693 & .870 & .921 & .718 & .270 & .417 \\
$\begin{array}{l}P \\
\text { exacta }\end{array}$ & .898 & .699 & $.019^{*}$ & .401 & .606 & .387 & 1.000 & .056 & .699 & .898 & .949 & .748 & .277 & .424 \\
\hline
\end{tabular}

Tabla 3

Indicadores estructurales de la rejilla en función de la presencia (n=13)-ausencia (n=11) de dilemas

\begin{tabular}{|c|c|c|c|c|c|c|c|c|c|}
\hline & $\begin{array}{l}\text { Intensi- } \\
\text { dad }\end{array}$ & Potencia & Polaridad & $\begin{array}{l}\text { Indefini- } \\
\text { ción }\end{array}$ & CFI & $\begin{array}{l}\text { R Yo- } \\
\text { Ideal }\end{array}$ & $\begin{array}{l}\text { R Yo- } \\
\text { otros }\end{array}$ & $\begin{array}{l}\text { R Ideal- } \\
\text { Otros }\end{array}$ & PVEPE \\
\hline U & 57.000 & 44.000 & 50.000 & 66.500 & 58.500 & 67.000 & 51.000 & 50.000 & 37.000 \\
\hline Z & -.840 & -1.593 & -1.246 & -.290 & -.755 & -.261 & -1.188 & -1.246 & -1.999 \\
\hline $\begin{array}{l}\text { P asin } \\
\text { tótica }\end{array}$ & .401 & .111 & .213 & .772 & .450 & .794 & .235 & .213 & $.046^{*}$ \\
\hline P exacta & .424 & .119 & .228 & .776 & .459 & .820 & .252 & .228 & $.047^{*}$ \\
\hline
\end{tabular}

rejilla.

En la tabla 2 se pueden ver los resultados relativos a la asociación entre dilemas y psicopatología. Sólo se encuentran diferencias en función de los dilemas en la escala de Sensibilidad Interpersonal. Para esta variable el rango promedio correspondiente al grupo sin dilemas es $8.27(\mathrm{M}=33.45, \mathrm{SD}=92.278)$ y el grupo con dilemas $14.73(M=215.82 ; \mathrm{SD}=152.196)$. $\mathrm{La}$ relación entre dilemas y variables estructurales de la rejilla muestra (tabla 3 ) que únicamente el porcentaje de varianza explicada por el primer eje arroja diferencias entre ambos grupos. El grupo con dilemas tiene un rango promedio de 15.64 (Media 43.598, SD 10.463) y el que no presenta dilemas de $9.85(\mathrm{M}=49.243, \mathrm{SD}=11.677)$.

Para finalizar el apartado de resultados se indican los estadísticos descriptivos de las variables que han sido introducidas en las hipótesis y/o han obtenido diferencias significativas en los análisis no paramétricos anteriores (tabla 4).

Cómo se puede ver, los valores de la polaridad de la muestra son altos para los dos grupos (se considera alta una polaridad por encima de 30) y el PVEPE es también alto, por encima de 40 en ambos grupos y pasando de 48 en la muestra de alta sintomatología (ver Feixas, de la Fuente y Soldevila, 2003). El porcentaje de presencia de dilemas es del 50\%, siendo ligeramente más alto en el subgrupo de alta sintomatología. El grupo de baja sintomatología muestra un porcentaje mucho mayor $(81.81 \%)$ de correlaciones positivas (teniendo en cuenta simplemente el signo de la puntuación) entre el yo y el ideal que el grupo de alta sintomatología $(46.15 \%)$.

\section{Discusión}


Tabla 4

Descriptivos de las variables introducidas en las hipótesis para los subgrupos de alta y baja sintomatología según la Dicotomización del IGS

\begin{tabular}{|c|c|c|c|c|c|c|}
\hline \multirow{2}{*}{ x Variables } & \multicolumn{2}{|c|}{$\begin{array}{l}\text { Muestra total } \\
(\mathrm{N}=24)\end{array}$} & \multicolumn{2}{|c|}{$\begin{array}{l}\text { Alta Sintomatología } \\
\qquad(\mathrm{N}=13)\end{array}$} & \multicolumn{2}{|c|}{$\begin{array}{l}\text { Baja Sintomatología } \\
(N=11)\end{array}$} \\
\hline & Media & $D T$ & Media & $D T$ & Media & $D T$ \\
\hline Polaridad & 41.925 & 17.120 & 46.735 & 19,741 & 36.242 & 11.869 \\
\hline Indefinición ** & 8.203 & 9.476 & 5.060 & 5.951 & 11.919 & 11.662 \\
\hline $\mathrm{CFI} \mathrm{F}^{* *}$ & .387 & .217 & .313 & .184 & .474 & .229 \\
\hline PVEPE & 46.185 & 11.165 & 48.732 & 10.486 & 43.176 & 11.677 \\
\hline $\begin{array}{l}\text { Sensibilidad Interpersonal } \\
\text { SCL }^{* *}\end{array}$ & 174.640 & 129.853 & 177.910 & 77.503 & 171.360 & 171.405 \\
\hline Ideación Paranoide SCL & 165.360 & 86.706 & 194.730 & 101.716 & 136.000 & 59.537 \\
\hline R Yo_Ideal & .156 & .366 & .050 & .343 & .282 & .368 \\
\hline $\begin{array}{l}\text { Porcentaje de relaciones } \\
\text { positivas yo-ideal }\end{array}$ & $58.33 \%$ & - & $46.15 \%$ & - & $81.81 \%$ & - \\
\hline $\begin{array}{l}\text { Porcentaje de presencia } \\
\text { de dilemas }\end{array}$ & $50 \%$ & - & $53.80 \%$ & - & $45.54 \%$ & - \\
\hline
\end{tabular}

Nota. ** Diferencias significativas

El número de resultados objetivos es bastante escaso, lo que indica que solo unas pocas variables de la rejilla son sensibles a las diferencias en sintomatología. Estas variables son la indefinición, el número de constructos funcionalmente independientes (CFI) y el porcentaje de varianza explicado por el primer eje (PVEPE). Si se ponen en relación los resultados con las hipótesis formuladas, se debe concluir que la hipótesis 1 ha quedado claramente infirmada, ya que no se encuentran diferencias en polaridad entre mujeres con alta y baja sintomatología en ninguno de los tres indicadores sintomatológicos estudiados. La hipótesis 2 queda confirmada parcialmente, ya que en el caso del IGS y del Inventario de Depresión de Beck se comprueba que el grupo con menos intensidad de la sintomatología muestra un mayor valor del número de constructos funcionalmente independientes, pero no se encuentran diferencias significativas en el otro indicador de complejidad, el PVEPE. La hipótesis 3, relacionada con la mayor presencia de psicopatología en las mujeres dilemáticas recibe un apoyo muy parcial, ya que sólo se encuentran diferencias significativas en el caso de las escalas del SCL relacionadas con el juicio social (sensibilidad interpersonal). Soldevilla et al. (2009) encuentran indicadores de que las mujeres maltratadas presentan menores valores de complejidad (un alto valor en PVEPE) y altos valores en polaridad cuando se comparan con mujeres no afectadas por la violencia. Igualmente, encuentran importantes discrepancias en las distancias entre el yo y el ideal, siendo las mujeres víctimas las que más se alejan de su idealización. Este fenómeno también se ha encontrado en mujeres abusadas en su infancia (Harter, 2000).

No obstante, algunos resultados no difieren esencialmente de los encontrado en estudios previos, más bien matizan los resultados. La polaridad de ambas submuestras es muy elevada, lo que concuerda con los datos ofrecidos por Garcia-Martínez et al. (2009) y Soldevilla et al. (2009), es decir, las mujeres maltratadas constituyen un grupo de alta polaridad, carac- 
terizado por una construcción extremada de su mundo social, algo que es coherente con las vivencias de amenaza a la que se ven sometidas, ya que una construcción polarizada permite una acción más clara, aunque ésta no sea eficaz. Las poblaciones con alto neuroticismo, como es el caso de todos los que pueden manifestar en mayor o menor medida problemas afectivos, suelen manifestar altos valores de polaridad (Winter, 1992).

Un resultado similar es el que se encuentra en el caso de la presencia-ausencia de dilemas. Si tenemos en cuenta que el porcentaje de dilemas en muestras normales es del 33\% y en muestras con patologías se acerca al 66\% (Feixas y Saúl, 2004; Saúl, 2006), la muestra total de este estudio se mueve entre ambas cifras. En cualquier caso, no se puede esperar en todas las mujeres maltratadas una tendencia general a mostrar dilemas, ya que muchas de ellas, especialmente si están en tratamiento, son capaces de definir claramente que la fuente de su malestar es el agresor (Walker, 2004), lo que disolvería las paradojas cognitivas en las que consisten los dilemas. No obstante, la traumatización es por sí misma una fuente de dilemas y obstáculos cognitivos (Sewell, 1987), lo que hace esperable que puedan aparecer dilemas en un porcentaje relativamente alto de mujeres maltratadas.

Por otro lado, la presencia de dilemas genera una útil diferencia en la sintomatología. Las mujeres con dilemas puntúan más en sensibilidad interpersonal y muestran una tendencia a un mayor perfil paranoide. Es decir, son más sensibles al juicio social y a elaborar un concepto negativo y perverso de los otros. Es verdad que este perfil puede tener incluso una cierta aura protectora, de autodefensa, cuando alguien se encuentra en peligro objetivo, ya que maximiza las posibilidades de detectar un posible problema. Pero el hecho de que se dé fundamentalmente en mujeres que presentan dilemas hace pensar en una estructura más neurótica de la personalidad centrada en la elaboración paradójica de las relaciones interpersonales. $\mathrm{Pa}$ rece que son las mujeres que presentan dilemas a las que les costará más construir sus relaciones con terceros. No hay evidencia de resultados de este tipo en la investigación anterior, pero abre una línea sugerente de trabajo en la resolución de dilemas presentes en las víctimas.

Los resultados relativos a la complejidad concuerdan con lo encontrado en otros estudios, en el sentido de que a mayor problematicidad, menor complejidad. La muestra es, en general, poco compleja si atendemos a los valores del PVEPF que son altos en los dos subgrupos y el subgrupo con alta sintomatología presenta además menores valores de índice CFI, lo que implica que no sólo es un grupo de baja diferenciación (Soldevilla et al, 2009), sino que además tiende a la simplicidad (Feixas y Cornejo, 2002). Queda por resolver el problema de si ambas medidas de complejidad miden o no lo mismo (complejidad cognitiva) o si una es más sensible que otra a la diferenciación, es decir a la falta de conexión entre unos constructos y otros. El problema de una medida exacta de la integración-diferenciación no está bien resuelto en la literatura sobre constructos personales, a pesar de las diferentes propuestas realizadas (Winter, 1992; Gallifa y Botella, 2000). En cualquier caso, los resultados de nuestro estudio indican que la tendencia a mostrar menores valores de CFI es mayor a medida que aumenta la sintomatología. El grupo que presenta mayor número de dilemas, es a su vez menos complejo y más monolítico en términos del indicador PVEPE. Esto parece indicar que la combinación de alta sintomatología con la presencia de dilemas genera un perfil con la mayor simplicidad posible, tanto en términos de agrupaciones de constructos como de dimensiones de significado.

Otro resultado interesante es el que muestra que las mujeres con menor intensidad de la sintomatología muestran mayores índices de indefinición. La indefinición es conceptualmente contraria a la polaridad, pero refleja el hecho de no poder tomar decisiones, de ser incapaz de anticipar acciones concretas. Si este fuera el caso, el perfil cuadraría con el de una víctima claramente controlada por su agresor, es decir, que se encuentra en un momento alto del proceso de dominación (Walker, 2004). Pero, en nuestra muestra, las mujeres con menor indefinición reflejan menos sintomatología, lo que parece indicar que son menos rígidas y más adaptativas. 
Resultados menos concordantes con investigaciones de otros autores son los relacionados con la distancia entre el yo y el ideal. La muestra de mujeres de este estudio presenta una relativamente buena correlación entre el yo y el ideal, a diferencia de lo encontrado por otros autores (Harter, 2000; Soldevilla et al., 2009). No obstante hay que tener en cuenta que es el subgrupo con menor intensidad de la sintomatología el que desvía claramente estos resultados hacia la concordancia entre ambos aspectos del self. Posiblemente este fenómeno de distanciamiento del yo ideal se da sobre todo en las mujeres con un alto grado de victimización.

Llama la atención que no se hayan producido resultados concordantes en cuanto a la estructura del sistema cognitivo con la dicotimización hecha a través de la prueba IAB. Es posible que se trate de un instrumento no adecuado para valorar los niveles de ansiedad de las mujeres maltratadas o bien que la sintomatología depresiva tenga mayor valor diferenciador que la ansiosa para esta muestra.

En definitiva, los resultados de este estudio matizan los encontrados en estudios similares que comparan mujeres víctimas con mujeres sin este perfil. Parece que la intensidad de la sintomatología es un factor a tener en cuenta en el modo en que se construye la estructura cognitiva de la personalidad. En este trabajo se han comparado dos subtipos de mujeres afectadas por la violencia contra la pareja, por lo que las diferencias entre ellas no deben ser tan drásticas como las que se encuentran con mujeres no afectadas por la violencia. En cualquier caso, los resultados indican que es necesario tener en cuenta diferentes tipologías de las víctimas.

En general, las tipologías de víctimas diferencian varios subtipos en función del tipo de afectación, pudiéndose diferenciar entre mujeres resilientes y no resilientes al proceso de victimización (Humphreys, 2003) y mujeres que presentan o no síntomas de estrés postraumáticas (Bàguena, Beleña, Roldán, Díaz y Villarroya, 2006). En diversos estudios anglosajones, las tasas de sintomatología depresiva en mujeres víctimas de violencia contra la pareja varían ente el 23 y el $64 \%$ y la sintomatología postraumática entre el 33 y el 88\%. (Warshaw y Barnes,
2003). Estos resultados parecen indicar que entorno a un $20 \%$ de las mujeres maltratadas por sus parejas no genera una sintomatología clínicamente relevante, lo cual va en la dirección de los estudios de la psicología positiva que indican que un importante grupo de personas sometidas a un trauma aprende a enfrentarse al mismo y no sucumbe a sus efectos (Tedeschi y Calhoun, 2001). Los estudios sobre menores afectados por la violencia de género muestran también la existencia de diversas tipologías que abarcan a menores resilientes, con sintomatología de tipo afectivo intenso, con sintomatología afectiva moderada y con indicadores postraumáticos (Graham-Bermann y Perkins, 2010), una distribución que también se ha encontrado en mujeres maltratadas de entornos rurales (GarciaMartínez, 2006).

Posiblemente, el subgrupo de baja sintomatología está más próximo a las mujeres resilientes o, al menos, a un grupo de sintomatología moderada. Estos primeros resultados parecen indicar que cada tipología de mujeres afectadas por la violencia puede construir de forma diferente su estructura cognitiva. Ello hace pensar que futuras líneas de investigación debería orientarse a evaluar de una manera explícita los niveles de resiliencia y de estrés postraumático, además de la sintomatología general y ansiosodepresiva para poder configurar de una manera más precisa las diferentes tipologías. Una vez hecho esto, sería posible pergeñar procesos terapéuticos diferenciados para cada subgrupo a partir de las variables de la rejilla. Obviamente en este diseño habría que incluir también variables de tipo cualitativo como los contenidos de los constructos (Feixas, Geldschläger y Neimeyer, 2002) y el modo en que se configuran agrupaciones de elementos (Garcia-Martínez, Orellana-Ramírez y Guerrero-Gómez, 2010).

Por último, indicar algunas de las deficiencias de este estudio. Sería necesario disponer de una muestra mayor, así como de una muestra de contraste de mujeres no afectadas por la violencia de características demográficas similares, para poder contrastar diferencias no sólo entre subtipos de víctimas. Además, sería recomendable ampliar el estudio a mujeres de extracción urbana, ya que es sabido que la violencia contra la pareja en el ámbito rural tiene 
claras características diferenciadoras como una mayor co-ocurrencia de diferentes tipos de abusos, un mayor invisibilización de la violencia y una menor disponibilidad de recursos frente a la misma (Logan, Walker, Cole, Ratliff y Leukefel, 2003; Teaster, Roberto y Dugar, 2006).

\section{Referencias}

Amor, P. J., Echeburúa, E., Corral, P., Zubizarreta, I. y Sarasua, B. (2002). Repercusiones psicopatológicas de la violencia doméstica en la mujer en función de las circunstancias del maltrato [The psychopathological consequences of domestic violence in women in function of maltreatment circumstances]. Revista Internacional de Psicología Clínica y de la Salud, 2, 227-246.

Bàguena, M. J., Beleña, A., Rodán, C., Díaz, A. y Villarroya, E. (2006). Mujeres maltratadas con y sin estrés post-traumáticos: análisis diferencial. En P. J. Costa, C. M. Lopes Pires, J. Veloso y C. T. Lopes Pires (Coords.), Stresse pós-traumático. Modelos, abordagenes e práticas (pp. 71-80). Leiria, Portugal: Diferença.

Bannister, D. y Fransella, F. (1966). Grid test of thought disorder. Barnstaple, Reino Unido: Psychological Tests Publications.

Beck, A. T., Brown, G., Epstein, N. y Steer, R. A. (1988). An inventory for measuring clinical anxiety: psychometric properties. Journal of Consulting and Clinical Psychology, 56, 893-897. doi:10.1037//0022-006X.56.6.893.

Beck, A. T., Rush, A. J., Shawn, B. F. y Emery, G. (1979). Cognitive therapy of depression. New York: Guilford Express.

Bobes, J., García-Portilla, M. P.; Bascarán, M. T., Sáiz, P. A. y Bousoño, M. (2004). Banco de instrumentos básicos para la práctica de la psiquiatría clinica [Bank of Basic Instuments to the Practice of Clinic Psychiatry]. Barcelona, España: Psiquiatría.

Buchbinder, E. y Eisikovits, Z. (2003). Battered women's entrapment in shame. A phenomenological study. American Journal of Orthopsichiatry, 73, 355-366. doi: 10.1037/0002-9432.73.4.355.

Camps, A., Calle, S. y Feixas, G. (2000). La construcción del maltrato en la violencia familiar cronificada [Maltreatment Construction in Cronic Family Violence]. Redes. Revista de Psicoterapia Relacional e Intervenciones Sociales, 6, 41-51.

Derogatis, L. R. (2002). Cuestionario de 90 Sintomas. Manual (J. L. González de Rivera, C. de las Cuevas, M. Rodríguez Abuín y F. Rodríguez-Pulido, Trad.). Madrid, España: TEA. 
Dutton, D. G. y Painter, S. L. (1981). Traumatic bonding: the development of emotional attachments in battered women and other relationships of intermittent abuse. Victimology: An International Journal, 6,139-155.

Echeburúa, E. y de Corral, P. (1998). Manual de violencia familiar [Family Violence Handbook]. Madrid, España: Siglo XXI.

Feixas, G. y Cornejo, J. M. (2002). RECORD v. 4.0: Análisis de correspondencias de constructos personales [Correspondence Analysis of Personal Constructs] [programa informático]. Barcelona, España: Psimedia. Recuperado de www.terapiacongitiva.net/record.

Feixas, G. y Cornejo, J. M. (1996). Manual de la técnica de la rejilla mediante el programa Record [Grid Tecnique Manual using the Gricord Software]. Barcelona, España: Paidós.

Feixas, G., de la Fuente M. y Soldevila, J. M (2003). La técnica de la rejilla como instrumento de evaluación y formulación de hipótesis clínicas [Grid Technique as Instrument to Assessment and Formulation of Clinical Hypotheses]. Revista de Psicopatología y Psicología Clínica, 6, 153-172.

Feixas, G., Geldschläger, H. y Neimeyer, R. A. (2002). Content analysis of personal constructs. Journal of Constructivist Psychology, 15, 1-19. doi:10.1080/107205302753305692.

Feixas, G. y Saúl, L. A. (2004). The Multi-Center Dilemma Project: An Investigation on the Role of Cognitive Conflicts in Health. The Spanish Journal of Psychology, 7(1), 69-78.

Feixas, G., Saúl, L. A., y Avila, A. (2009). Viewing cognitive conflicts as dilemmas: Implications for mental health. Journal of Constructivist Psychology, 22, 141-169.

Feixas, G., Saúl, L. A., Avila, A. y Sánchez Rodríguez, V. (2001). Implicaciones terapéuticas de los conflictos cognitivos [Therapeutic implications of cognitive conflicts]. Revista Argentina de Clínica Psicológica, $X(1)$, 5-13.

Fischbach, R. L. y Herbert, B. (1997). Domestic violence and mental health: correlates and conundrums within and across cultures. Social Science and Medicine, 45, 1161-1176. doi: 10.1016/S02779536(97)00022-1
Fransella, F., Bell, R. y Bannister, D. (2004). A Manual for Repertory Grid Technique. Second Edition. Chichester, Reino Unido: Wiley.

Gallifa, J. y Botella, L. (2000). The structural quadrants method: a new approach to the assessment of contructs system complexity via the repertory grid. Journal of Constructivist Psychology, 13, 1-26.

Garcia-Martínez, J. (2006). Violencia contra la mujer y estrés postraumático [Violence Against Women and Post-Traumatic Stress]. En P. J. Costa, C. M. Lopes Pires, J. Veloso y C. T. Lopes Pires (Coords.), Stresse pós-traumático. Modelos, abordagenes e práticas (pp. 81-90) [Post-traumatic stress: models, approaches and practices]. Leiria, Portugal: Diferença.

Garcia-Martínez, J. (2008). La conciencia del otro: agresores y víctimas desde una perspectiva constructivista [The Awareness of the Other: Offenders and Victims from a Constructivist Approach]. Apuntes de Psicología, 26, 361-378.

Garcia-Martínez, J., Cano-García, F. J., RodríguezFranco, L., Buero-Gallego, M., Alcocer-Gómez, E. y Guerrero-Gómez, R. (2009). Linking Traits and Personal Constructs: An Exploratory Study throughout Levels of Personality. Annals of Psychology, 12, 57-80.

Garcia-Martínez, J., Orellana-Ramírez, M. C. y Guerrero-Gómez, R. (2010). La construcción de la identidad y de los roles de género en mujeres maltratadas: un estudio a través de la técnica de la rejilla [Identity construction and gender roles in battered women: a study using the grid technique]. En I. Vázquez (Coord.), Investigaciónes Multidisciplinares en género. Actas del II Congreso Universitario Nacional de Investigación y Género (pp. 297314) [Gender Multidisciplinary Researchers: Minutes of the 2nd National University Congress on Research and Gender]. Sevilla, España: Digital@3.

Golding, J. M. (1999). Intimate partner violence as a risk factor for mental disorders: A meta-analysis. Journal of Family Violence, 14, 99-132.

Graham-Bermann, S. A. y Perkins, S. (2010). Effects of early exposure and lifetime exposure to intimate partner violence (IPV) on child adjustment. Violence and Victims, 25, 427-439. doi: 10.1891/0886-6708.25.4.427.

Guerrero-Gómez, R., Garcia-Martínez, J. y OrellanaRamírez (2009). Construcción del Maltrato e In- 
tensidad de la Sintomatología [Maltreatment Construction and Symptoms Intensity]. En S. N. de Jesus (Coord.), Actas do I Congresso LusoBrasileiro de Psicologia da Saúde (pp. 1085-1102). Faro, Portugal: Universidade Do Algarve.

Harter, S. L. (2000). Quantitative mesaures of constructing in child abuse survivors. Journal of Constructivist Psychology, 13, 103-116.

Heise, L. y Garcia Moreno, C. (2002). Violence by intimate partners. En E. G. Krug, L. L. Dahlberg, J. A. Mercy, A. B. Zwi y R. Lozano (Eds.), World report on violence and health. Ginebra, Suiza: Organización Mundial de la Salud.

Horley, J. y Johnson, A. (2008). Meaning and change with domestic abusers. En J. D. Raskin y S. K. Bridges (Eds.), Studies in meaning, 3: Constructivist Psychotherapy in the real world (pp. 127144). Nueva York: Pace University Press.

Humphreys, J. (2003). Resilience in sheltered battered women. Issues in Mental Health Nursing, 24, 137-152.

Jacobson, N. S. y Gottman, J. (1998). When men battered women. Nueva York: Simon y Schuster.

Kelly, G. A. (1955). The Personal Constructs Psychology. Nueva York: Norton.

Koss, M. P., Koss, P. G. y Woodruff, W. J. (1991). Deleterious effects of criminal victimization on women's health and medical utilization. Archives of Internal Medicine, 151, 342-347. doi: 10.1001/archinte.151.2.342.

Krug, E. G., Dahlberg, L. L., Mercy, J. A., Zwi, A. B. y Lozano, R. (Eds.). (2002). World report on violence and health. Ginebra, Suiza: Organización Mundial de la Salud.

Logan, T. K., Walker, R., Cole, J., Ratliff, S. y Leukefel, C. (2003). Qualitative Differences Among Rural and Urban Intimate Violence Victimization Experiences and Consequences: A Pilot Study Journal of Family Violence, 18, 83-92.

Matud, P. (2004). Impacto de la violencia doméstica en la salud de la mujer maltratada [The impact of domestic violence on the health of abused women]. Psicothema, 16, 397-401.
Orava, T. A., McLeod, P. J. y Sharpe, D. (1996). Perceptions of control, depressive symptomatology, and self-esteem in women in transition from abusive relationships. Journal of Family Violence, 11, 167-186. doi:10.1007/BF02336668.

Römkens, R. (1997). Prevalence of wife abuse in the Netherlands. Journal of Interpersonal Violence, 12, 99-125. doi: 10.1177/088626097012001007.

Saúl, L. A. (2006). El papel de los conflictos cognitivos en la salud mental: Implicaciones para el cambio terapéutico [Role of Cognitive Conflict in Mental Health: Implications to Therapeutic Change] (Tesis Doctoral). Salamanca, España: Universidad de Salamanca.

Sewell, K.W. (1987). Posttraumatic Stress: Towards a Constructivist Model of Psychotherapy. En G. J. Neimeyer y R. A. Neimeyer (Eds.), Advances in Personal Constructs Psychology (Vol. 4, pp. 207235). Greenwich, CO: JAI Press.

Soldevilla, J. M., Feixas, G., Varlotta, N., Cirici, R. y Ayats, M. (2009, julio). Structural characteristics of the constructs systems of women victims of gender violence. Comunicación presentada en el XVIIIth International Congress on Personal Construct Psychology.

Teaster, P. B., Roberto, K. A. y Dugar, T. A. (2006). Intimate partner violence of rural aging women. Family Relations, 55, 636-648. doi:10.1111/j.17413729.2006.00432.x.

Tedeschi, R. G. y Calhoun, L. G. (2000). Posttraumatic growth: A new focus in traumatology. Psytalk, Newsletter of the British Psychological Society Student Membres Group, April.

Walker, L. (2004). Abused women and survivor therapy. Washington, DC: American Psychological Association.

Warshaw, C. y Barnes, H. (2003). Domestic Violence, Mental Health y Trauma. Research Higlights. The domestic Violence and Mental Health Policy Iniciative, Chicago, Ill, April.

Winter, D. A. (1992). Personal Constructs Psychology in clinical practice: Theory, Research and Applications. Londres, Reino Unido: Routledge. 